\title{
A VLBA Calibrator Survey
}

\section{A. B. Peck ${ }^{1,2}$ and A. J. Beasley ${ }^{2}$}

Abstract. The Very Long Baseline Array (VLBA) Calibrator Survey consists of snapshot observations at 2.7 and $8.4 \mathrm{GHz}$ of nearly 2000 flat-spectrum extragalactic sources selected from the Jodrell Bank-VLA Astrometric Survey (JVAS). When complete, this survey will assist in defining the extragalactic radio frame in the northern sky by providing positions for these sources which will be accurate on the milliarcsecond scale. This survey is accessible on the WWW at http://magnolia.nrao.edu/vlba_calib/index.html.

Phase-referencing in VLBI is useful in extending coherent integration times and achieving precise astrometric measurements. This technique requires rapid switching between the target source and a nearby calibrator. The VLBA Calibrator Survey will provide milliarcsecond-accurate positions for $\sim 2000$ calibrator sources which can be used for this purpose. In addition to the astrometry, there will be numerous scientific returns related to the imaging of these data. When completed, this survey will have increased by an order of magnitude the number of radio sources observed with milliarcsecond resolution and component spectral-index information. Using the Caltech DIFMAP package (Shepherd, Pearson, \& Taylor 1994), these data are being imaged to search for compact steep-spectrum and symmetric objects, milliarcsecond gravitational lenses (millilenses), and should provide exciting new data for $\mu-z$ and $\mu-\theta$ studies when the JVAS optical follow-up (Patnaik et al. 1992) is complete. Studies of scattering as a function of Galactic latitude and longitude should also be possible. Many new and unique objects will be identified for the first time by this survey.

VLBA Calibrator Survey Sources

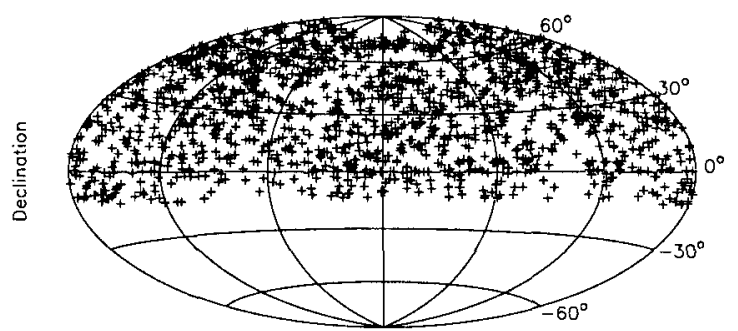

Right Ascension

Figure 1. Sources in the 8 epochs of the VLBA Calibrator Survey which have been observed as of June, 1997.

The northern sky has been divided into 8 regions by declination. The regions above $0^{\circ}$ declination have now been imaged. All of the sources observed in the survey to date are shown in Figure 1. The survey itself is available on the World Wide Web at http://magnolia.nrao.edu/vlba_calib/index.html. The

\footnotetext{
${ }^{1}$ Dept. of Physics, New Mexico Tech, Socorro, NM 87801, USA

${ }^{2}$ National Radio Astronomy Observatory, Socorro, NM 87801, USA
} 
list of calibrators can be searched either by IAU source name or by J2000 coordinates. The results of a search may be sorted by source name, by distance from the center of the search radius, or by peak flux at X-band. Each search yields a table containing the IAU name of the source, the J2000 coordinates, and the peak X-band flux. In addition, the image and visibility plots for most sources, (examples shown in Figures $2 \mathrm{a}$ and $2 \mathrm{~b}$ ), are available. The search by coordinates will also produce a diagram of the proximity of sources found to the search center, as shown in Figure 2c. This diagram can be printed or saved to a PostScript file, along with the accompanying table.
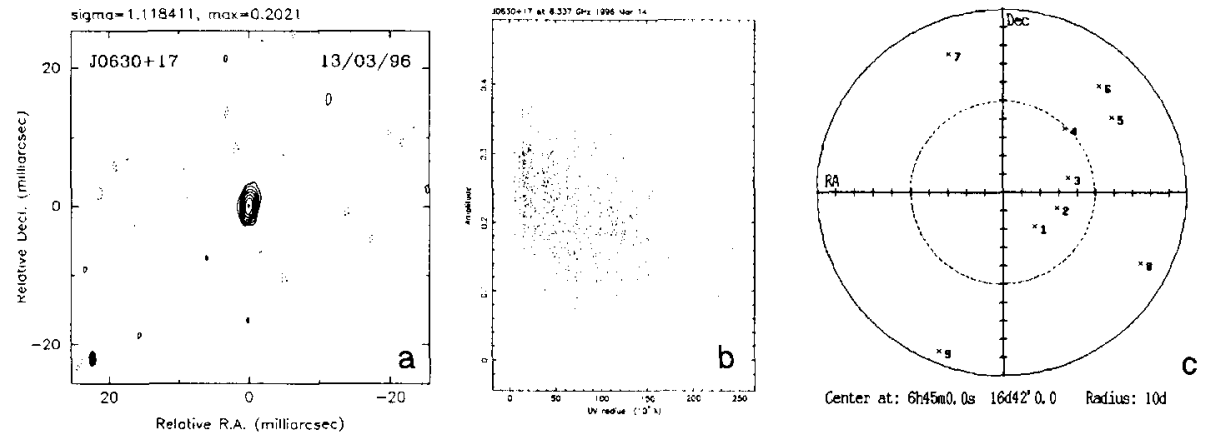

Figure 2. (a) A sample source returned by the search centered on coordinates (06h45m0s $\left.16 \mathrm{~d} 42^{\prime} 0.0^{\prime \prime}\right)$; (b) The visibility plot of the $8.4 \mathrm{GHz}$ data for the same source; (c) The proximity diagram generated by searching by coordinates.

Several updates to the VLBA Calibrator Survey can be expected. The remaining region (declinations $0^{\circ}$ to $-14^{\circ}$ ) will be imaged. Declinations $-14^{\circ}$ to $-30^{\circ}$ and $79^{\circ}$ to $90^{\circ}$ will be observed. Also, another observing run will be scheduled to observe sources which were either missed or contained bad data on the first run. In the case of a few very weak sources, $(\leq 60 \mathrm{mJy})$, it appears that the images generated by the DIFMAP scripts used have artifacts created by DIFMAP in the absence of real data. These sources will be further studied to determine if any of them can be reobserved to obtain more reliable data. Maps made by the DIFMAP scripts which appear to be particularly noisy will be reviewed and imaged individually where possible. Final astrometric processing of all epochs is expected by January 1998.

Acknowledgments. We wish to thank Stèphane Bèland for creating the prototype search engine and web page. AP was supported for much of this research by the New Mexico Space Grant Consortium. The National Radio Astronomy Observatory is a facility of the National Science Foundation, operated under a cooperative agreement by Associated Universities, Inc.

\section{References}

Patnaik, A. R., et al. 1992. MNRAS, 254, 655-676.

Shepherd, M. C., Pearson T. J., \& Taylor, G. B. 1994. BAAS, 26, 987-989. 